\title{
USO DEL TIEMPO LIBRE EN EL CANTON DE TURRIALBA Y SU INFLUENCIA EN LA CALIDAD DE VIDA SEGÚN EDAD Y GÉNERO
}

\author{
Olman Vargas Zumbado \\ Escuela de Educación Física y Deportes \\ Universidad de Costa Rica, San José, Costa Rica \\ E-mail:ovargasz@cariari.ucr.ac.cr
}

\begin{abstract}
Resumen
Vargas-Zumbado, O. (2006). Uso del tiemp libre en el cantón de Turrialba y su influencia en la calidad de vida según edad y génerl. Revista de Ciencias del Ejercicio y la Salud, 4(1), 52-60. Se pretende determinar cómo utilizaban el tiempo libre en el Cantón Central de Turrialba; cuál fue su influencia en la calidad de vida según edad y género; identificar las actividades que se realizan según edad y género y si éstas contribuyen a la calidad de vida, en las relaciones familiares y en el aspecto físico, social y psicológico, finalmente, si la población joven es más activa que el adulto o el adulto mayor. Se determinó que los (as) participantes cuentan con tiempo libre para recrearse y que los hombres cuentan con más tiempo libre que las mujeres. Ambos géneros opinan que es importante la recreación para el bienestar físico, social y psicológico y que les ayuda a mejorar su calidad de vida. La tendencia independientemente del género fue a escoger actividades recreativas pasivas (ver televisión, escuchar música y dormir), en casi todos los grupos de edades, solamente los hombres entre los 30 a 39 años escogieron caminar como actividad preferida, luego ver televisión y dormir. PALABRAS CLAVES: Tiempo libre, actividades recreativas, edad, género, calidad de vida.
\end{abstract}

\section{INTRODUCCIÓN}

Durante varios años se ha hablado sobre la importancia del uso del tiempo libre y sus beneficios en diferentes estratos sociales, así como sobre los problemas que aquejan a las personas para poder disponer de tiempo para su recreación y buscar estilos de vida saludables en función de una mejor calidad de vida.

El presente artículo tiene como objetivo principal analizar la situación que se vive en el cantón Central de Turrialba con respecto al uso del tiempo libre, caracterizar las actividades que se realizan y establecer las preferencias según género y edad, así como determinar si los jóvenes son más activos que los adultos.

El estudio de la Recreación en Costa Rica, según Gutiérrez (1989), data de 1930 como Geografía de la Recreación, y las primeras políticas en Costa Rica se presentan durante la época de los 70, específicamente en 1974; sin embargo, el tema del uso del tiempo libre y la recreación se retoma nuevamente a finales de los 90. Morera y Rojas (2000) reportan los datos recolectados para la zona de Talamanca y San Vito de Coto Brus, los cuales, junto con esta investigación y otras que se piensan realizar por ejemplo: Uso del tiempo libre en la población estudiantil de las diferentes Sedes de la Universidad de Costa Rica, pueden contribuir a la formación de un perfil sobre la situación actual a nivel nacional.

El autor de esta investigación define al tiempo libre, como el tiempo del que se dispone fuera de las tareas del trabajo, o del horario que se dedica a las labores hogareñas o al estudio, es decir, el tiempo en que se puede realizar la actividad que le plazca a la persona, tal como leer, ver televisión, jugar, escuchar música, tejer, bailar, pintar, escribir, en fin, toda aquella actividad que pueda producir placer y que no arriesgue la salud de 
la persona o de las que la rodeen. Es importante, además, determinar de cuánto tiempo libre dispone la población; sabemos que las necesidades actuales han aumentado progresivamente, por lo que es usual que las personas necesiten dedicarle más tiempo al trabajo o que incluso desempeñen más de un trabajo para satisfacer las demandas familiares de casa, comida, vestido, servicios básicos o servicios médicos, entre otras. En contra posición, también se dice que cada día queda más tiempo libre porque la jornada laboral ha ido cambiando.

Al respecto, Ruiz, García y Hernández (2001), luego de un estudio descriptivo, en la Universidad de Almería ( España) con jóvenes universitarios en una muestra de más de 2000 estudiantes, encontraron que era muy importante dedicar tiempo libre a la práctica físico-deportiva como una de las formas ideales $y$ de mayor diversidad de manifestaciones entre las actividades de ocio. Invernó (1997) por su parte presenta como una alternativa de ocio y recreación el montar en bicicleta, y presenta un programa integral para favorecer el uso del tiempo libre en forma segura, ordenada $\mathrm{y}$ eficiente para mejorar la salud y la calidad de vida. También, Guirao (2000) presenta los beneficios del buen uso del tiempo libre asociado a la discapacidad psíquica realizando actividades lúdico-deportivas como fuente de disfrute y experiencia gozosa, y cómo estas les ayudan a desarrollar hábitos y actitudes de convivencia, cooperación, independencia, autonomía, y desarrollo motriz, y a abandonar el sedentarismo y superar el aislamiento, la obesidad y la falta de relaciones interpersonales. Zea, (2000) presenta un planteamiento de cómo experimentar el uso del tiempo libre desde una perspectiva educativa-recreativa de la mano de la aceptación de la diversidad considerando diferentes discapacidades, y concluye que todas las personas diferentes han de tener la misma oportunidad que el resto de la sociedad para poder disfrutar del tiempo libre. Morera y Rojas (2000), con una muestra de 176 jóvenes entre los 14 y 16 años de las regiones de la Reserva Bribrí-Cabécar en Talamanca y de San Vito de Coto Brus, concluyeron de que la actividad deportiva que más practicaban en su tiempo libre era jugar fútbol; escribir fue la actividad cultural de preferencia y la actividad social más frecuente fue salir con amigos; la actividad recreativa preferida fue escuchar música; ir al culto o a misa fue la actividad religiosa seleccionada, y prefieren disfrutar de su tiempo libre en compañía de otras personas como amigos, novia(o), algún familiar u otras personas. También encontraron diferencias significativas en cuanto al uso del tiempo libre entre hombres y mujeres, así como en el disfrute del mismo y el permiso que deben solicitar. Vargas (2003), encontró que en general la tendencia tanto en hombres como en mujeres fue de realizar actividades recreativas en su mayoría de tipo pasivas, lo cual es preocupante si se analiza desde el punto de vista físico, porque se estaría cayendo en el sedentarismo, lo cual va en contra de la salud y del principio actual de buscar estilos de vida saludable.

\section{METODOLOGÍA}

\section{Participantes:}

Los sujetos de investigación fueron 122 personas entre los 18 y 62 años residentes en el Cantón de Turrialba de la Provincia de Cartago. De ellos 63 fueron hombres y 59 mujeres.

De los hombres 30 (47\%) eran solteros, $29(46 \%)$ casados, 1 (1.58\%) viudo, 1 $(1.58 \%)$ divorciado, 1 (1.58\%) en unión libre y $1(1.58 \%)$ no respondió. Con respecto a la profesión u oficio, de ellos $16(25.39 \%)$ eran estudiantes, 4 (6.35\%) profesores, 4 (6.35\%) comerciantes, $3(4.76 \%)$ oficinistas, 3 (4.76\%) contadores, $3(4.76 \%)$ mecánicos, 3 (4.76\%) pensionados, $3(4.76 \%)$ ingenieros, 3 $(4.76 \%)$ dependientes y $21(33.33 \%)$ se distribuyeron en otras actividades. En cuanto al nivel educativo, 27 hombres (43\%) tenían estudios universitarios completos, 2 (3.17\%) estudios universitarios incompletos, 21 
(33.33\%) con secundaria completa, 3 (4.76\%) con secundaria incompleta y $10(16 \%)$ con primaria completa.

Por otro lado, el ingreso económico promedio de los que trabajaban fue de ciento cuarenta mil ochocientos veinticuatro colones.

La información de las mujeres se presenta seguidamente: $30 \quad(51 \%)$ eran solteras, 23 (39\%) casadas, 2 (3.38\%) viudas, $3(5 \%)$ divorciadas y $1(1.7 \%)$ no respondió. En relación con el oficio o profesión, 21 $(36 \%)$ eran estudiantes, $10(17 \%)$ amas de casa, 8 profesoras $(14 \%), 5$ comerciantes $(8.5 \%), 4$ secretarias $(7 \%), 9 \quad(15 \%)$ desempeñaban otros oficios y 2 (3.38\%) no respondieron. En cuanto al nivel educativo, $26(44 \%)$ contaban con educación universitaria completa, 1 incompleta (2\%), 22 $(37 \%)$ con secundaria completa y $1(2 \%)$ incompleta, 7 (12\%) con primaria completa y $2(3.38 \%)$ incompleta.

El ingreso económico promedio de las que trabajaban fue de ciento dieciocho mil novecientos cincuenta colones mensuales.

Se seleccionó inicialmente una muestra de 200 sujetos por un procedimiento no probabilístico intencional por algunos de los vecindarios cercanos al centro de Turrialba. De esa muestra se consideraron 63 hombres y 59 mujeres que devolvieron el cuestionario.

\section{Instrumento de medición}

Se confeccionó un instrumento de medición luego de analizar varios de ellos. Dicho cuestionario se adaptó a las actividades propias del país y se validó por juicio de expertos (3 profesionales en el Campo de la Educación Física y con experiencia en Programas de Recreación lo revisaron), y luego de los ajustes se procedió a administrarlo.

El instrumento consta de un apartado de introducción sobre el objetivo del

Cuestionario. Además, se les presentó una definición de tiempo libre dentro del encabezado (Considere tiempo libre como aquel en que usted se dedica a realizar actividades fuera del trabajo o fuera de las labores hogareñas, es decir, fuera de las obligaciones cotidianas).

Posteriormente, se adjudicó un espacio para los datos personales: edad, sexo, estado civil, número de hijos, nivel educativo (completo o incompleto) profesión u oficio, ingresos económicos. Luego se suministró la instrucción de marcar con (X) la respuesta que considerara que se ajustaba más a su situación $\mathrm{y}$ finalmente, 10 preguntas relacionadas con el uso del tiempo libre, calidad de vida, autoimagen (apariencia física) y el estado anímico general de los sujetos de investigación.

\section{Procedimiento}

Para la recolección de la información colaboraron estudiantes residentes de la Sede del Atlántico. Se les instruyó para que repartieran el cuestionario en el área cercana a su casa, únicamente a aquellas personas mayores de 18 años y que distribuyeran equitativamente el $50 \%$ hombres y $50 \%$ mujeres.

NOTA: Para efectos de este artículo cuyo principal fin fue comparar el tipo de actividades recreativas, según género y edad se dividió a los sujetos de la siguiente manera:

Mujeres en cuatro grupos entre las siguientes edades: 18 a 29 años; 30 a 39 años; 40 a 50 años.

Hombres en cuatro grupos entre las siguientes edades: 18 a 29 años; 30 a 39 años; 40 a 62 años.

Además, que si el sujeto decía que esperara de una vez para responder el cuestionario, debían esperar.

\section{Análisis Estadístico}

Se obtuvo la estadística descriptiva (frecuencias absolutas y relativas) y se analizó por medio de gráficos de frecuencias. 


\section{RESULTADOS}

En términos generales se pudo determinar que tanto hombres como mujeres independientemente del la edad, indicaron lo siguiente:

Que disponían de tiempo libre para dedicarse a realizar actividades recreativas; que se sentían bien con su apariencia física y autoimgen, que sienten que las actividades recreativas les ayudan a tener: una aceptable calidad de vida, mejoran las relaciones familiares y les ayuda a sentirse bien física, psicológica y mentalmente.

En cuanto a las preferencias de las actividades recreativas según género y edad, se presenta la información de la siguiente forma: en el Gráfico $\mathrm{N}^{0}$ 1, las actividades Recreativas realizadas pòr las mujeres entre 18 y 29 años de edad; mientas que en el Gráfico $\mathrm{N}^{\circ} 2$, se puede apreciar las Actividades Recreativas realizadas por los hombres de la misma edad.

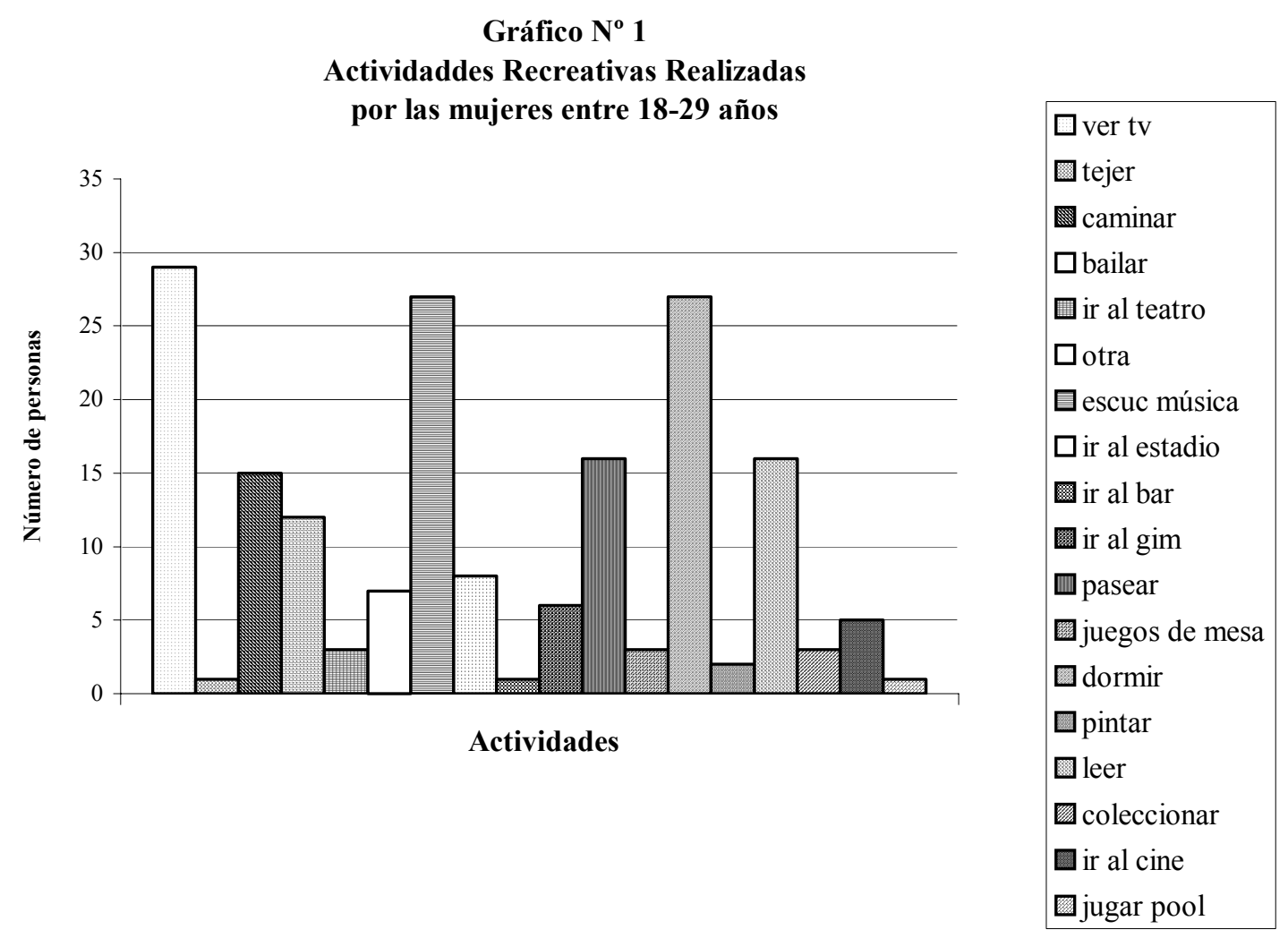

Como se aprecia en los Gráficos 1 y 2 , tanto hombres como mujeres en este grupo de edades, coinciden en la preferencia de las actividades recreativas, en su mayoría escogieron: ver televisión, escuchar música y dormir.
Mientras tanto, en los Gráficos 3 y 4, se puede apreciar que las personas entre los $30 \mathrm{y}$ los 39 años: las mujeres en orden descendente escogieron: ver televisión, caminar y dormir. Los hombres en primer lugar escogieron caminar, ver televisión y dormir. 


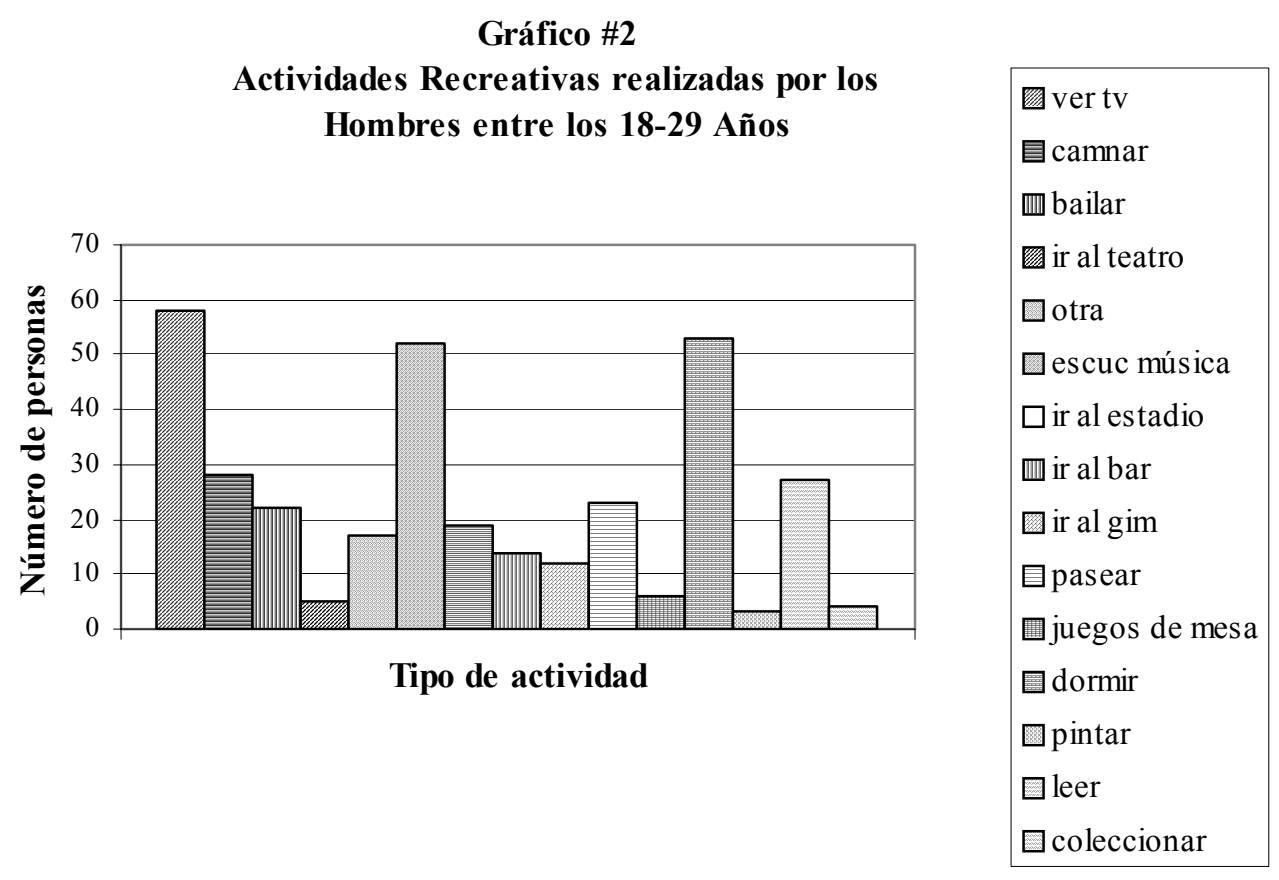

Gráfico No3

Actividades Recreativas Realizadas por las mujeres entre 30-39 Años

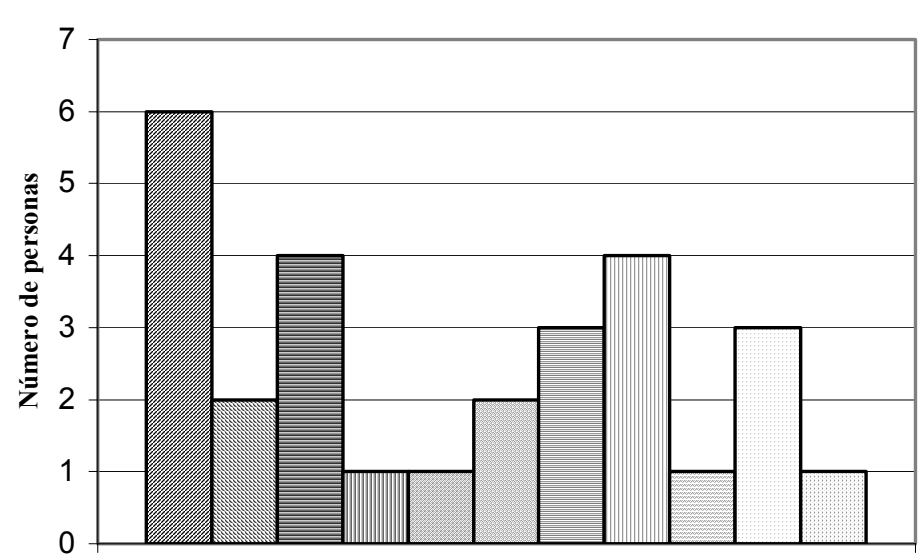

$\square$ ver tv

$\square$ bailar

$\square$ caminar

四 ir al gim

口otra

$\square$ pasear

目escuc música

$\square$ dormir

$\square$ pintar

$\square$ leer

Tipo de actividad

$\square$ ir al cine 
Gráfico $\mathbf{N}^{\mathbf{0}} \mathbf{4}$

Actividades Recreativas realizadas por los

Hombres entre los 30-39 Años

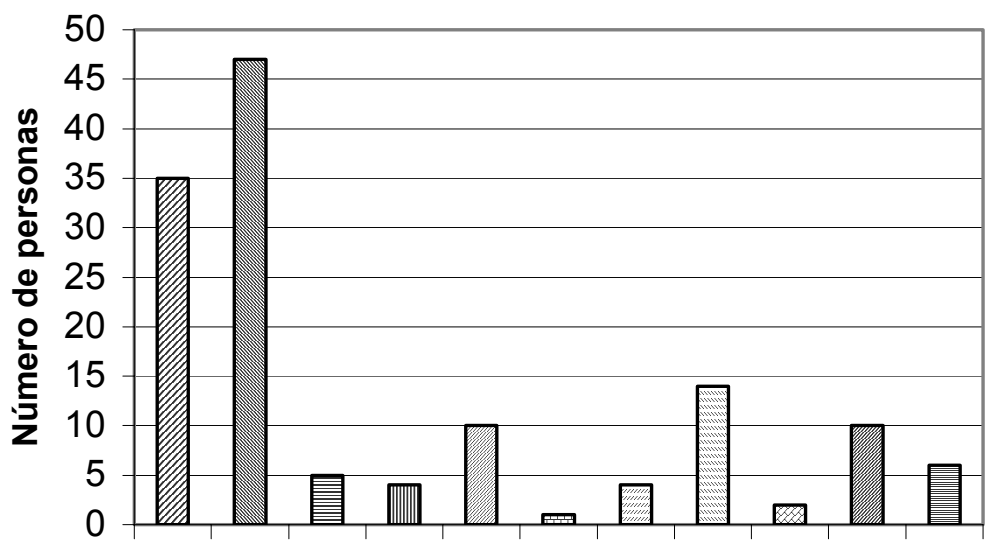

\begin{tabular}{|c|}
\hline ver tv \\
\hline$\square$ camnar \\
\hline 目bailar \\
\hline 皿 otra \\
\hline$\square$ escuc música \\
\hline 圈 ir al estadio \\
\hline Oir al bar \\
\hline dormir \\
\hline 国pintar \\
\hline leer \\
\hline 目pasear \\
\hline
\end{tabular}

Tipo de Actividad

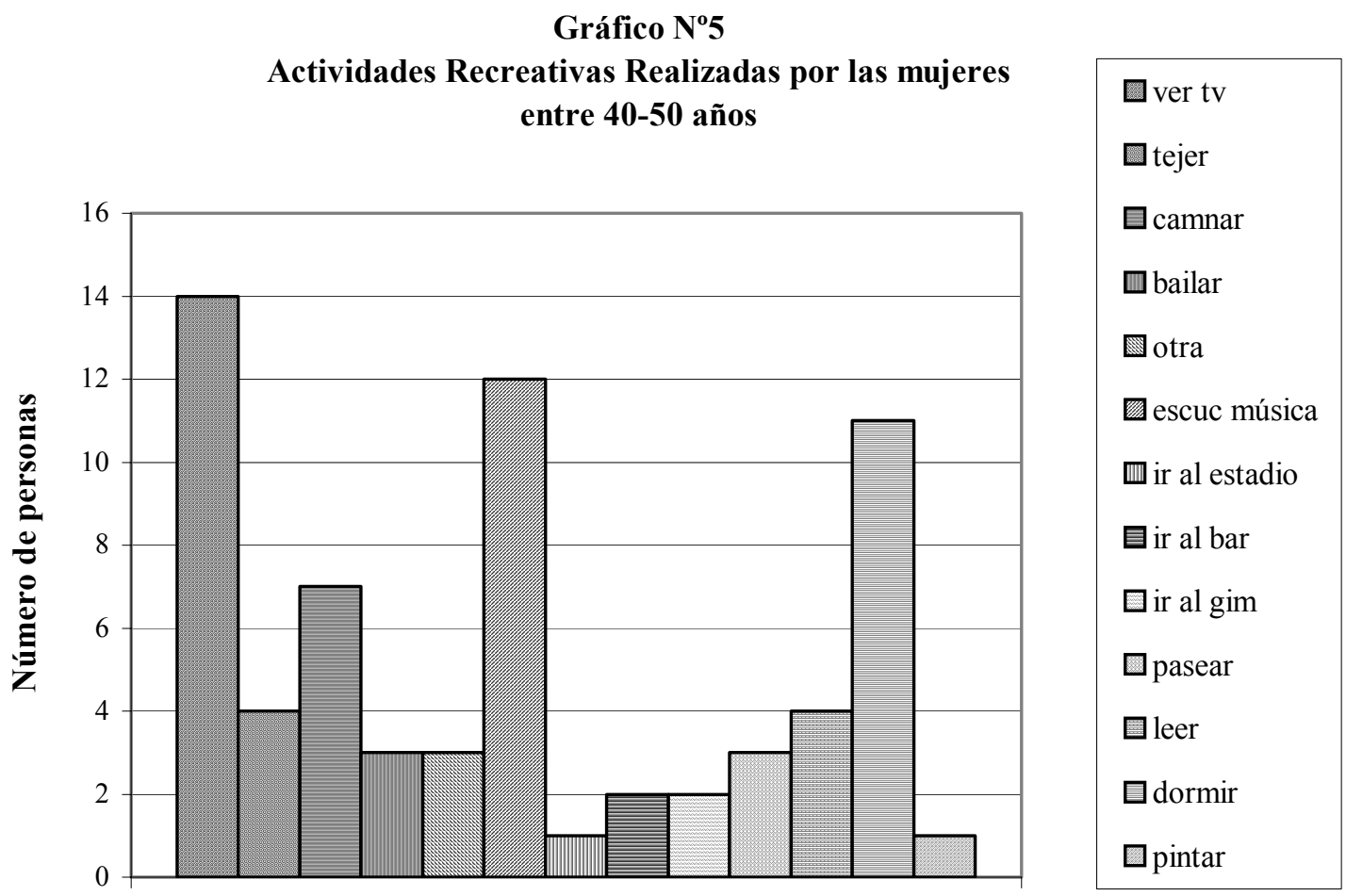

Tipo de âctividad 
En el grupo de edades de 40 a 62 años, las mujeres escogieron preferentemente: ver televisión, escuchar música y dormir, mientras los hombres seleccionaron: ver televisión, escuchar música y caminar

Gráfico $\mathbf{N}^{\mathbf{0} 6}$

Actividades Recreativas realizadas por los

Hombres entre los 40-62 Años

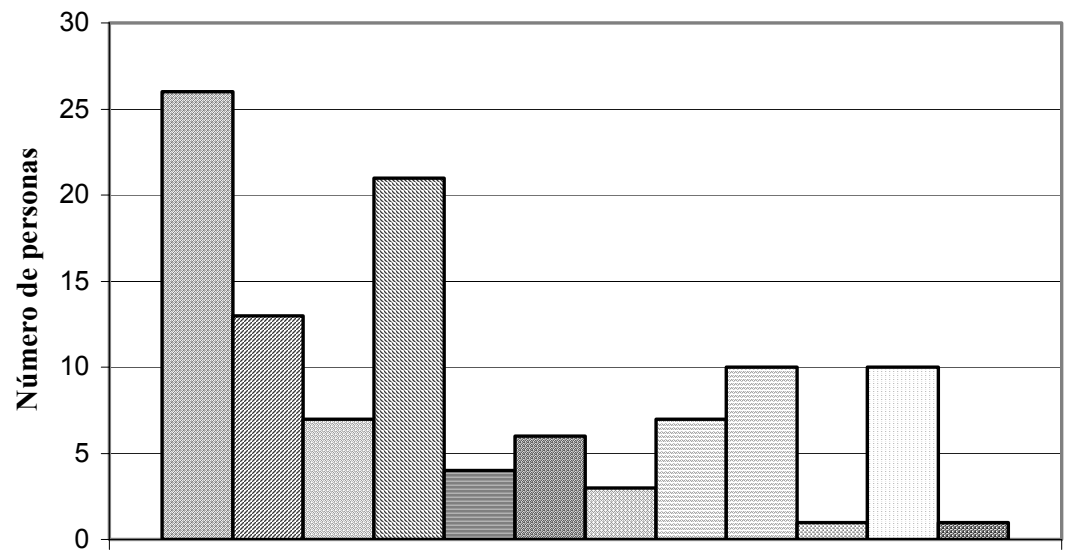

Tipo de actividad

\begin{tabular}{|l}
$\square$ ver tv \\
$\square$ camnar \\
$\square$ otra \\
$\square$ escuc música \\
$\square$ ir al estadio \\
$\square$ ir al bar \\
$\square$ ir al gim \\
$\square$ pasear \\
$\square$ dormir \\
$\square$ pintar \\
$\square$ leer \\
$\square$ pool
\end{tabular}

\section{DISCUSIÓN}

Con base en la información recopilada se desprende que en el Cantón Central de Turrialba existe un criterio generalizado, tanto en hombres como en mujeres, de la importancia de dedicarse al uso del tiempo y la recreación ( $76 \%$ y $64.4 \%$ respectivamente), como un medio para liberar tensiones y estrés, y para sentirse mejor anímicamente y físicamente (hombres $87 \%$ y mujeres $100 \%$ ), lo cual contribuye a una mejor percepción de su apariencia física (hombres $87 \%$, mujeres $70 \%$ ); también les ayuda a mejorar las relaciones familiares y a disfrutar con sus amigos (hombres $87 \%$ y mujeres 100\%). Además, al realizar actividades recreativas se sienten mejor, más saludables física $\mathrm{y}$ psicológicamente, los mantiene ocupados en actividades sanas, se sienten felices, autorrealizados y consideran que mejoran su calidad de vida, además, de rendir mejor en el trabajo. Estos resultados coinciden con los encontrados por Morera y Rojas (2000), Cañellas y Rovira (1995), Ponce de León (1998), Ruiz-García y Hernández (2001), Zea (2000), Gracia, Marcó y Garre (1999), Guirao (2000) y Mora (2002) y Gutiérrez (1989).

En cuanto a las actividades recreativas que más practicaban en el centro de Turrialba, según género y edad, se encontró que tanto los hombres como las mujeres tienen una tendencia a escoger actividades pasivas (ver televisión, escuchar música y dormir). Se presentó una diferencia el grupo de edades entre 30 a 39 años, los hombres escogieron en primer lugar caminar y luego ver televisión y dormir, mientras que las mujeres seleccionaron, ver televisión, caminar en segundo lugar y dormir. Lo anterior, rompe el patrón que se venía presentando en que escogieron actividades 
pasivas. Tal vez se puede atribuir a que en ese grupo de edades la mayoría laboraban como docentes y comerciantes, lo cual podría sugerir que tal vez disponían de más tiempo, recursos económicos, conciencia y madurez de la importancia de realizar actividades recreativas más vigorosas, lo cual también se relaciona con la opinión de sentirse mejor con la apariencia física y su estado emocional, para ese grupo de edades. En las edades de 40 a 50 años (62 años mujeres) en las dos primeras actividades no hubo diferencias (escogieron ver televisión $\mathrm{y}$ escuchar música), los hombres en tercer lugar escogieron caminar, mientras que las mujeres escogieron dormir.

Estos hallazgos fueron diferentes a los que inicialmente se esperaban, es decir, que los jóvenes tanto hombres como mujeres prefirieran actividades más dinámicas como, caminar, ir al gimnasio, bailar, participar en deportes. También, difieren de lo encontrado por Gutiérrez (1989), en donde la actividad preferida en el Gran Área Metropolitana fue ir a la playa y visitar parques en segundo lugar. El deporte de preferencia fue la natación, seguido de la gimnasia $\mathrm{y}$ el atletismo en el tercer lugar. Esto posiblemente se deba al nivel educativo tanto de hombres como mujeres, que tal vez les ha permitido tomar conciencia de la realidad económica y de la importancia de compartir en familia, además, del gran impacto e influencia de los medios de comunicación colectiva como la televisión, los problemas de seguridad ciudadana y las limitaciones económicas, entre otras.

Los resultados obtenidos concuerdan en parte con lo encontrado por Morera y Rojas (2000), Gutiérrez (1989), Vargas (2003), en lo referente a la importancia de compartir tanto en familia o grupos de edades afines, y que era vital como un medio de salud y recreación.

Es preocupante lo encontrado en esta investigación, porque la tendencia fue a escoger actividades recreativas pasivas, lo que se concluye que la televisión, escuchar música y dormir ocupan un lugar muy importante en dicho Cantón, independientemente del grupo de edades, lo cual genera la duda con respecto a la parte de la salud física. Se espera investigar posteriormente dicho aspecto.

\section{CONCLUSIONES}

Luego del análisis se llega a las siguientes conclusiones:

1- Los (as) participantes en la investigación dicen contar con tiempo libre para dedicarlo a realizar actividades recreativas. Tienen mayor cantidad de tiempo libre los hombres que las mujeres

2- Ver televisión, escuchar música y dormir son las actividades de preferencia tanto en hombres como en mujeres, independientemente de la edad, únicamente el patrón cambió en los hombres en las edades entre los 30 y 40 años, en donde tienden a escoger una actividad dinámica como caminar. Todas las actividades seleccionadas parecen favorecer las relaciones familiares, y dijeron sentirse bien y que mejoran su calidad de vida.

3- Existe aceptación tanto por parte de los hombres como de las mujeres en recibir capacitación; sin embargo, las mujeres dicen tener más disponibilidad que los hombres.

4- Tanto hombres como mujeres indicaron que es muy importante dedicarle tiempo a realizar actividades recreativas.

5- También, existe acuerdo entre géneros y grupos de edades de la importancia de la recreación y su influencia en el bienestar físico, social y psicológico y que les ayuda a tener una mejor calidad de vida.

6- Con respecto a la apariencia personal (imagen corporal), los hombres están más conformes que las mujeres, independientemente de la edad.

7- En cuanto al estado anímico, los hombres tienen mejor estado de ánimo que las mujeres, principalmente entre los 30 a los 39 años, posiblemente porque una mayoría le gusta caminar. 


\section{REFERENCIAS}

Cañellas, A. (1995). Los hábitos deportivos de la población adulta barcelonesa (15 a 59 años) Rev.Apunts, Educación Física y Deportes 42:75-79.

Gracia, M; Marcó, M. y Garre, J. (1999). Valoración de los beneficios psicológicos del ejercicio en personas mayores. Rev.Apunts, Educación Física y Deportes 57:46-54.

Guirao, I. (2000).Ocio y discapacidad psíquica. Rev.Apunts: Educación Física y Deportes 60:66-69.

Gutiérrez, R. (1989). Los espacios Recreativos del Gran Área Metropolitana. Tesis de Licenciatura Escuela de Historia y Geografia. Universidad de Costa Rica.

Hernádez, S.; Fernández, C. y Baptista, P. (2003) Metodología de la Investigación. 3 ed. México: McGraw Hill Interamericana de México, S.A.

Invernó, J. (1997). Circulemos en bicicleta. Una propuesta interdisciplinar de educación viaria. Rev.Apunts, Educación Física y Deportes 48: 92-101.

Mora, J. (2002). Aplicación de un programa de ejercicios aeróbicos en personas mayores, resultados obtenidos tras valoración ergométrica. Ponencia IX Simposio Internacional de Ciencias del Ejercicio y la Salud. San José, Costa Rica.

Morera, M y H. Rojas. (2000). Comparación del uso del tiempo libre en los y las jóvenes de 14 a 16 años en las regiones de la Reserva Indígena de Talamanca y San Vito de Coto Brus. Tesis de Grado. Universidad Nacional. Costa Rica.

Ponce de León, A. (1998). Análisis de la Educación Física desde la perspectiva de una Educación para el Tiempo Libre. Rev.Apunts, Educación Física y Deportes 51:23-34.

Ruiz, F; M.García y A.Hernández (2001) El interés por la práctica de actividad físico-deportiva de tiempo libre del alumnado de la Universidad de Almería. Un estudio Longitudinal. Rev.Apunts: Educación Física y Deportes 63:86-92.

Vargas, O. (2003) Uso del tiempo libre en el Cantón de Turrialba y su influencia en la calidad de vida según género. Rev. Reflexiones Vol.82 \#1 2003

Zea, M. (2000).El ocio, tiempo para todos: planteamiento de un programa recreativo en torno a la dis-capacidad. Rev.Apunts: Educación Física y Deportes 60:70-75. 